\title{
The Resultant Iteration for Determining the Stability of a Polynomial*
}

\section{By James Lucien Howland}

\begin{abstract}
The square root iteration $x_{i+1}=\left(x_{i}+x_{i}^{-1}\right) / 2$ is known to be convergent to +1 from starting values $x_{0}$ in the open right half-plane, and to -1 from starting values in the open left half-plane. The resultant of the quadratic $z^{2}-2 w z+1$ and a given polynomial $P(z)$, of proper degree $n$, is a polynomial $R(w)$ whose zeros, expressed in terms of the zeros $z_{i}$ of $P(z)$, are just $w_{i}=\left(z_{i}+z_{i}^{-1}\right) / 2$. The construction of $R(w)$ is thus equivalent to $n$ independent applications of the square root iteration, with unknown starting values $z_{i}$. Repetition of this construction generates a sequence of resultants whose zeros are independently convergent to either +1 or -1 according as the initial zero of $P(z)$ lies in the open right or left half-plane. When the given polynomial, and each of the resultants, is written as a linear combination of the $n+1$ polynomials $(z+1)^{n},(z+1)^{n-1}(z-1),(z+1)^{n-2}(z-1)^{2}, \ldots,(z+1)(z-1)^{n-1}$, $(z-1)^{n}$. the sequence of resultants is ultimately convergent to one of the elements $(z+1)^{n-p}(z-1)^{p}$ of this basis, whence it follows that $P(z)$ has $p$ zeros with positive real part and $n-p$ zeros with negative real part, and the Hurwitz stability problem for $P(z)$ is solved. By an application of the principle of argument it is, in general, possible to determine $p$ at a finite stage of the resultant iteration.

When formulated in terms of this basis, the resultant iteration becomes formally identical with the root-squaring process. This fact may be exploited to establish the properties of the resultant iteration, and to show that root-squaring may be applied to solve the Schur stability problem for a given polynomial. Although precise results are not available, numerical results and the general properties of this iteration suggest that, for the purpose at hand, it is unaffected by the progressive deterioration of condition that sometimes occurs in other applications of root-squaring.
\end{abstract}

1. Introduction. The two stability problems for a polynomial-the first, that of determining the numbers of its zeros with positive, zero, and negative real parts, and the second, that of determining the numbers of its zeros within, on, and without the unit circle-are important in connection with the determination of the stability of linear differential and difference equations, respectively. Numerical methods for solving these problems must be simpler and more efficient than algorithms for evaluating all the zeros of a polynomial, since both stability problems are solved when the numerical values of the zeros are known. This paper is concerned with an iterative procedure, called the resultant iteration, for the solution of either of these stability problems which, to the extent that iterative procedures can be contrasted, appears to be more efficient than available methods for computing all the zeros.

Received August 16, 1977 ; revised October 28, 1977. $70 \mathrm{~K} 15$.

AMS (MOS) subject classifications (1970). Primary 12D10; Secondary 34D20, 65H05,

*This research was supported, in part, by the National Research Council of Canada, Grant A3028. 
The results reported here were motivated by the properties and applications of the matrix sign function [5], which suggest the possibility of constructing an analogous "polynomial sign function". Such a polynomial would have zeros \pm 1 , distributed accordingly as a given polynomial of proper degree $n$, with which it is associated, has zeros with positive or negative real part. The "polynomial sign function" would, thus, be one of the $n+1$ polynomials $(z+1)^{n-j}(z-1)^{j}, 0 \leqslant j \leqslant n$, namely $(z+1)^{n-p}(z-1)^{p}$, whenever the given polynomial has $p$ zeros with positive real parts. Such a polynomial might be computed by an appropriate adaptation of the iteration

$$
S(i+1)=\left[S(i)+S^{-1}(i)\right] / 2, \quad S(0)=A,
$$

used to calculate the sign matrix associated with a given matrix $A$.

The resultant procedure, developed and investigated by E. H. Bareiss [1], [2] in connection with root-squaring, may be adapted to the computation of polynomials analogous to the matrices $S(i)$. For, the resultant $R(w)$ of a given polynomial $P(z)$ and the quadratic polynomial

$$
Q(z)=z^{2}-2 w z+1
$$

vanishes when, and only when, $P(z)$ and $Q(z)$ have a common factor. Thus, if $P(z)=$ $Q(z)=0$, it follows from (2) that

$$
w=\left(z+z^{-1}\right) / 2,
$$

whence it is seen that the zeros of $R(w)$ are related to those of $P(z)$, respectively, by the formula (3), analogous to (1), which defines an iteration convergent to the square roots of 1 . Iteration of the resultant procedure, justified by the known convergence properties of the square-root iteration, thus supplies the required polynomials and, ultimately, the associated "sign polynomial".

The resultant procedure may be reformulated in terms of the polynomial basis $(z+1)^{n-j}(z-1)^{j}, 0 \leqslant j \leqslant n$, so that, once $P(z)$ and $Q(z)$ are written in terms of the elements of this basis, all the resultants are similarly expressed. Thus, if

$$
\begin{aligned}
P(z)= & a_{0}(z+1)^{n}+a_{1}(z+1)^{n-1}(z-1) \\
& +\cdots+a_{j}(z+1)^{n-j}(z-1)^{j}+\cdots+a_{n}(z-1)^{n},
\end{aligned}
$$

the resultant of $P(z)$ and $Q(z)$ is

$$
R(z)=A_{0}(z+1)^{n}+\cdots+A_{j}(z+1)^{n-j}(z-1)^{j}+\cdots+A_{n}(z-1)^{n},
$$

where

$$
A_{k}=(-1)^{k}\left[a_{k}^{2}+2 \sum_{i=1}^{n-k}(-1)^{i} a_{k-i} a_{k+i}\right], \quad 0 \leqslant k \leqslant n .
$$

If, now, there is an integer $p$ such that

$$
\left|A_{p}\right|>\sum_{j=0 ; j \neq p}^{n}\left|A_{j}\right|,
$$

then $(z+1)^{n-p}(z-1)^{p}$ and $R(z)$ have the same numbers of zeros in the open left and right half-planes. Since it may be shown that all the polynomials in the resultant 
sequence have the same numbers of zeros in these half-planes, it follows that the integer $p$ supplies the solution to the first stability problem for the given polynomial, and that $(z+1)^{n-p}(z-1)^{p}$ is the required "sign polynomial".

The formulae (6) define the root-squaring algorithm as applied to the polynomial

$$
a_{0} z^{n}+a_{1} z^{n-1}+\cdots+a_{j} z^{n-j}+\cdots+a_{n} .
$$

This fact may be developed to establish the properties of the resultant sequence, and to verify the fact, apparently not well known, that the root-squaring process can be applied to solve the second stability problem for a given polynomial. Thus, the investigation of polynomial resultant sequences leads to iterative algorithms for the solution of both the first and second stability problems.

These algorithms will clearly fail when, in the case of the first problem, the given polynomial has zeros lying exactly on the imaginary axis or, in the case of the second problem, the exceptional zeros lie on the unit circle. These cases are characterized by the failure of any single coefficient in the successive resultants to dominate, in the sense of the inequality (7). This possibility of failure, in turn, suggests the possibility of almost arbitrarily slow convergence whenever the given polynomial has zeros lying near, although not exactly on, the boundary separating the domains of convergence. In the case of the first problem, zeros with small real part, especially when associated with relatively large imaginary part, will give rise to slow convergence while, in the case of the second problem, zeros with modulus near 1 will have a similar effect.

When nonconvergence, or unacceptably slow convergence, is encountered, there seems to be little choice but to apply a direct or noniterative method, such as that of A. Cohn [4, Section I], which is formulated for the second problem, but may easily be adapted to the first.

2. The Calculation of the Resultants. To form the resultant of the polynomials

$$
P(z)=a_{0}(z+1)^{n}+\cdots+a_{j}(z+1)^{n-j}(z-1)^{j}+\cdots+a_{n}(z-1)^{n}
$$

and

$$
Q(z)=z^{2}-2 w z+1=-\frac{w-1}{2}(z+1)^{2}+\frac{w+1}{2}(z-1)^{2},
$$

Sylvester's dialytic method of elimination (see [3, Vol. 2, Section 154, p. 75]) may be adapted to obtain the resultant in the form of the $(n+2) \times(n+2)$ determinant

$$
R(w)=\operatorname{det}\left|\begin{array}{cccccccc}
a_{0} & a_{1} & a_{2} & a_{3} & \ldots & a_{n-1} & a_{n} & 0 \\
0 & a_{0} & a_{1} & a_{2} & \ldots & a_{n-2} & a_{n-1} & a_{n} \\
-(w-1) & 0 & w+1 & 0 & \ldots & 0 & 0 & 0 \\
0 & -(w-1) & 0 & w+1 & \ldots & 0 & 0 & 0 \\
0 & 0 & -(w-1) & 0 & \ldots & 0 & 0 & 0 \\
\cdot & . & . & . & \ldots & . & . & . \\
0 & 0 & 0 & 0 & \ldots & 0 & w+1 & 0 \\
0 & 0 & 0 & 0 & \ldots-(w-1) & 0 & w+1
\end{array}\right| .
$$


The important property of this function, for present purposes, is that $R(w)=0$ when, and only when, $P(z)$ and $Q(z)$ have a nonconstant common factor [14, p. 84].

By repeated application of the Laplace expansion it may be shown that $R(w)$ may be written

$$
R(w)=\sum(-1)^{i+j} \operatorname{det}\left|\begin{array}{cc}
a_{i} & a_{j+1} \\
a_{i-1} & a_{j}
\end{array}\right| B_{(j-i)}(w),
$$

where the sum is taken over all possible choices of the columns $i(0 \leqslant i \leqslant n)$, and $j(j \leqslant i ; 0 \leqslant j \leqslant n)$ of the determinant, and $B_{(j-i)}(w)$ is the product of the three minor determinants

$$
\operatorname{det}\left|\begin{array}{ccccc}
-(w-1) & 0 & w+1 & \ldots & 0 \\
0 & -(w-1) & 0 & \ldots & 0 \\
. & . & . & \ldots & . \\
0 & 0 & 0 & \ldots & -(w-1)
\end{array}\right|=[-(w-1)]^{i}
$$

of dimension $i$,

$$
\operatorname{det}\left|\begin{array}{cccccc}
0 & w+1 & 0 & \ldots & 0 & 0 \\
-(w-1) & 0 & w+1 & \ldots & 0 & 0 \\
0 & -(w-1) & 0 & \ldots & 0 & 0 \\
. & . & . & \ldots & . & . \\
0 & 0 & 0 & \ldots & -(w-1) & 0
\end{array}\right|=D_{j-i}
$$

of dimension $j-i$, and

$$
\operatorname{det}\left|\begin{array}{cccccc}
w+1 & 0 & 0 & \ldots & 0 & 0 \\
0 & w+1 & 0 & \ldots & 0 & 0 \\
-(1-w) & 0 & w+1 & \ldots & 0 & 0 \\
. & . & . & \ldots & . \\
0 & 0 & 0 & \ldots & 0 & w+1
\end{array}\right|=(w+1)^{n-j}
$$

of dimension $n-j$. The determinants $D_{j-i}(w)$ satisfy the recurrence formula

$$
D_{t}=(w-1)(w+1) D_{t-2} ; \quad D_{1}=0, \quad D_{0}=1,
$$

whence it follows that

$$
D_{j-i}(w)= \begin{cases}0 & \text { if } j-i \text { is odd } \\ {[(w-1)(w+1)]^{(j-i) / 2}} & \text { if } j-i \text { is even }\end{cases}
$$

(Similar expressions are discussed and illustrated by E. H. Bareiss [1, pp. 364, 365].) Thus 


$$
D_{j-i}(w)=\frac{1+(-1)^{j+i}}{2}[(w-1)(w+1)]^{(j-i) / 2}
$$

and, setting $k=(i+j) / 2$,

$$
R(w)=\sum(-1)^{j} \operatorname{det}\left|\begin{array}{cc}
a_{i} & a_{j+1} \\
a_{i-1} & a_{j}
\end{array}\right| \frac{1+(-1)^{2 k}}{2}(w+1)^{n-k}(w-1)^{k} .
$$

Noting that $i=2 k-j, 0 \leqslant k \leqslant n, k \leqslant j \leqslant n$, and defining $a_{i}=0$ for $i<0$ or $i>n$, this sum may be written

$$
\begin{aligned}
R(w) & =\sum_{k=0}^{n}\left[\sum_{j=k}^{n}(-1)^{j} \operatorname{det}\left|\begin{array}{cc}
a_{2 k-j} & a_{j+1} \\
a_{2 k-j-1} & a_{j}
\end{array}\right|\right](w+1)^{n-k}(w-1)^{k} \\
& =\sum_{k=0}^{n} A_{k}(w+1)^{n-k}(w-1)^{k},
\end{aligned}
$$

where the coefficients

$$
\begin{aligned}
A_{k} & =\sum_{j=k}^{n}(-1)^{j} \operatorname{det}\left|\begin{array}{cc}
a_{2 k-j} & a_{j+1} \\
a_{2 k-j-1} & a_{j}
\end{array}\right| \\
& =(-1)^{k}\left[a_{k}^{2}+2 \sum_{i=1}^{n-k}(-1)^{i} a_{k-i} a_{k+i}\right] .
\end{aligned}
$$

The formulae (5) and (6) are the means of constructing the consecutive elements of the resultant sequence, starting from a reformulation of $P(z)$ in terms of the polynomials $(z+1)^{n-j}(z-1)^{j}$. It should be noted that the formulae (6) define the rootsquaring process, a situation that will be seen to be perfectly natural when the properties of the square root iteration are developed below.

The root-squaring process has been extensively discussed and analyzed, with historical notes and references, by Bareiss [1], where the formulae (6) are derived.

3. The Conversion from Maclaurin Form. A polynomial $P(z)$, given in the conventional or Maclaurin form

$$
P(z)=b_{0} z^{n}+b_{1} z^{n-1}+\cdots+b_{n-1} z+b_{n}
$$

may be rewritten in terms of the polynomial basis $(z+1)^{n-j}(z-1)^{j}$ by a procedure described by Duffin [6, pp. 204-206] and, in a slightly different form, by Lanczos [8, pp. 37-39 and Appendix, pp. 509-512]. By setting

$$
z=(w+1) /(w-1)
$$

in $P(z)$, the rational function

$$
(w-1)^{-n}\left[b_{0}(w+1)^{n}+b_{1}(w+1)^{n-1}(w-1)+\cdots+b_{n}(w-1)^{n}\right]
$$


is obtained, whose numerator may be expressed in Maclaurin form to obtain

$$
(w-1)^{-n}\left[a_{0} w^{n}+q_{1} w^{n-1}+\cdots+a_{n}\right] .
$$

Now, the transformation (9) is involutory, so that

$$
w=(z+1) /(z-1), . \quad w-1=2 /(z-1),
$$

and, substituting these expressions, $P(z)$ is obtained in the required form

$$
P(z)=2^{-n}\left[a_{0}(z+1)^{n}+a_{1}(z+1)^{n-1}(z-1)+\cdots+a_{n}(z-1)^{n}\right] .
$$

The coefficients $a_{j}$ are, thus, those obtained when $P(z)$ is written in powers of $w$, under the transformation (9), and multiplied by $(w-1)^{n}$. They are linear combinations of the coefficients $b_{j}$ of $P(z)$, involving the binomial coefficients appearing in the expansions of $(w+1)^{n-j}(w-1)^{j}$ in powers of $w$.

An algorithm for the computation of the coefficients $a_{j}$ in terms of the coefficients $b_{j}$ may be adapted from the results obtained by Duffin (loc cit.) as follows:

(A) Define the matrix $\Gamma=\left(\Gamma_{i j}\right)$ by the generating functions

$$
(w+1)^{n-i}(w-1)^{i}=\sum_{j=0}^{n} \Gamma_{i j} w^{n-j}, \quad 0 \leqslant i \leqslant n .
$$

Then

$$
\begin{aligned}
P(z) & =\sum_{i=0}^{n} b_{i} z^{n-i}=(w-1)^{-n} \sum_{i=0}^{n} b_{i}(w+1)^{n-i}(w-1)^{i} \\
& =(w-1)^{-n} \sum_{i=0}^{n} \sum_{j=0}^{n} b_{i} \Gamma_{i j} w^{n-j}
\end{aligned}
$$

so that

$$
a_{j}=\sum_{i=0}^{n} b_{i} \Gamma_{i j}, \quad \text { or } \quad \mathrm{a}=\mathrm{b} \Gamma .
$$

(B) Note that the first row of $\Gamma$ is generated by the binomial $(w+1)^{n}$, and that the first column of $\Gamma$ consists entirely of 1 . Further, from the generating functions,

$$
(w+1)(w-1)^{-1} \sum_{j=0}^{n} \Gamma_{i j} w^{n-j}=\sum_{j=0}^{n} \Gamma_{i-1, j} w^{n-j},
$$

whence it is seen that

$$
\Gamma_{i, j+1}=\Gamma_{i-1, j+1}-\Gamma_{i j}-\Gamma_{i-1, j}
$$

and the entire matrix $\Gamma$ may be constructed from its first row and column by the algorithm described by the formula

$$
a=b-c-d
$$

and the array 
Related matrices are tabulated by Lanczos (loc. cit.) for $1 \leqslant n \leqslant 12$, and relevant coefficients are listed by Peltier [12, pp. 228-239] through $n=20$.

Thus, by performing the vector $\times$ matrix multiplication (11), the row vector $b$ is converted to the row vector a and, apart from the common scale factor $2^{n}$, the polynomial $P(z)$, given in the Maclaurin form (8), is converted to the form (4). This is a once-only computation, as the resultants subsequently obtained from (4) are all in this form.

It is well known that, via the mapping (9), the first and second stability problems for a given polynomial are entirely equivalent (see Duffin, loc. cit. p. 204). Thus, the investigation of the second stability problem may be undertaken by transforming the problem to the first stability problem for a related polynomial, and applying the resultant iteration. Since the transformation (9) is involutory, the first step in the solution of the first problem for the related polynomial is to apply the transformation (9), which will, apart from trivial scale factors, recover the coefficients of the original polynomial; only now as the coefficients of a polynomial in the form (4). Thus, to solve the second problem, this double transformation may be omitted entirely, and the root-squaring iteration, plus the termination criterion (7), applied directly to the Maclaurin coefficients of the given polynomial.

4. Termination of the Resultant Iteration. The following application of the principle of argument supplies a criterion for terminating the resultant iteration.

THEOREM. Suppose that

$$
R(z)=A_{0}(z+1)^{n}+\cdots+A_{j}(z+1)^{n-j}(z-1)^{j}+\cdots+A_{n}(z-1)^{n}
$$

and that, for some integer $0 \leqslant p \leqslant n$,

$$
\left|A_{p}\right|>\sum_{j=0 ; j \neq p}^{n}\left|A_{j}\right| .
$$

Then, $R(z)$ has exactly $p$ zeros with positive real part, and $n-p$ zeros with negative real part.

Proof. $R(z)$ may be written

$$
\begin{aligned}
R(z)=A_{p}(z+1)^{n-p}(z-1)^{p} & {\left[\frac{A_{0}}{A_{p}}\left(\frac{z+1}{z-1}\right)^{p}+\frac{A_{1}}{A_{p}}\left(\frac{z+1}{z-1}\right)^{p-1}+\cdots+1\right.} \\
& \left.+\frac{A_{p+1}}{A_{p}}\left(\frac{z+1}{z-1}\right)^{-1}+\cdots+\frac{A_{n}}{A_{p}}\left(\frac{z+1}{z-1}\right)^{p-n}\right] .
\end{aligned}
$$

According to the principle of argument (see [9, Theorems 1.2, 1.6, 1.3]), $R(z)$ and $(z+1)^{n-p}(z-1)^{p}$ have the same numbers of zeros in the open right and left halfplanes whenever

$$
\begin{aligned}
\mid \frac{A_{0}}{A_{p}}\left(\frac{z+1}{z-1}\right)^{p}+\cdots+\frac{A_{p-1}}{A_{p}}\left(\frac{z+1}{z-1}\right) & +\frac{A_{p+1}}{A_{p}}\left(\frac{z-1}{z+1}\right) \\
& +\cdots+\frac{A_{n}}{A_{p}}\left(\frac{z-1}{z+1}\right)^{n-p} \mid<1
\end{aligned}
$$


when $z$ lies on the imaginary axis $x=0$. In this case, however, $|(z+1) /(z-1)|=1$, and the inequality is satisfied whenever

$$
\left|\frac{A_{0}}{A_{p}}\right|+\cdots+\left|\frac{A_{p-1}}{A_{p}}\right|+\left|\frac{A_{p+1}}{A_{p}}\right|+\cdots+\left|\frac{A_{n}}{A_{p}}\right|<1,
$$

which is just the condition (7). Q.E.D.

This theorem was formulated, in the case of the unit circle and the second stability problem, by D.-E. Mayer [10, p. 111].

5. The Square Root Iteration. It follows from the formula

$$
z_{i+1}=\left(z_{i}+z_{i}^{-1}\right) / 2
$$

which defines the square root iteration, that

$$
\frac{z_{i+1}+1}{z_{i+1}-1}=\left(\frac{z_{i}+1}{z_{i}-1}\right)^{2}
$$

so that, if $\zeta_{i}=\left(z_{i}+1\right) /\left(z_{i}-1\right)$ is the image of $z_{i}$ under the conformal transformation

$$
\zeta=(z+1) /(z-1)
$$

then

$$
\zeta_{i+1}=\zeta_{i}^{2}
$$

The square root iteration is thus conformally equivalent to successive squaring. It may be concluded at once that

(A) If $z_{i}$ lies in the left/right half of the $z$-plane, $\zeta_{i}$ lies inside/outside the unit circle in the $\zeta$-plane. Then, $\zeta_{i+1}$ also lies inside/outside the unit circle, whence, since the transformation (13) is involutory, $z_{i+1}$ lies in the left/right half-plane.

(B) If $z_{i}$ lies on the imaginary axis in the $z$-plane, $\zeta_{i}$ lies on the unit circle in the $\zeta$-plane. Then, $\zeta_{i+1}$ lies on the unit circle and, as before, $z_{i+1}$ lies on the imaginary axis in the $z$-plane.

(C) If $z_{0}$ lies in the left/right half-plane, so that $\zeta_{0}$ lies inside/outside the unit circle, then $\left\{\zeta_{i}\right\}$ is convergent to $0 / \infty$ and $\left\{z_{i}\right\}$ is convergent to $-1 /+1$.

This last result is the justification for the entire resultant iteration, and shows that difficulties may arise when a zero of $P(z)$ is on, or near, the imaginary axis. It follows from (13) and (14) that

$$
\frac{z_{r}+1}{z_{r}-1}=\left(\frac{z_{0}+1}{z_{0}-1}\right)^{R}=\zeta_{0}^{R}
$$

where $R=2^{r}$, whence

$$
z_{r}-1=2 /\left(\zeta_{0}^{R}-1\right) ; \quad z_{r}+1=2 \zeta_{0}^{R} /\left(\zeta_{0}^{R}-1\right) .
$$

If $z_{0}$ lies in the left half-plane, so that $z_{r} \rightarrow-1$ and $\zeta_{0}^{R} \rightarrow 0$, then

$$
\left|z_{r}+1\right|=2\left|\zeta_{0}\right|^{R} /\left|\zeta_{0}^{R}-1\right| \leqslant 2\left|\zeta_{0}\right|^{R} /\left(1-\left|\zeta_{0}\right|^{R}\right)<\epsilon
$$


whenever $\left|\zeta_{0}\right|^{R}<\epsilon /(\epsilon+2)<\epsilon / 2$. Similarly, if $z_{0}$ lies in the right half-plane, so that $z_{r} \rightarrow+1$ and $\zeta_{0}^{R} \rightarrow \infty$, then

$$
\left|z_{r}-1\right|=2 /\left|\zeta_{0}^{R}-1\right| \leqslant 2 /\left(\left|\zeta_{0}\right|^{R}-1\right)<\epsilon
$$

whenever $\left|\zeta_{0}\right|^{-R}<\epsilon /(\epsilon+2)<\epsilon / 2$. Thus, the rate of convergence of the square root iteration is entirely dependent on the modulus of the initial value $\zeta_{0}$, and the rate of convergence of the resultant iteration is governed by the rate of convergence of the square root iteration with slowest convergence, where the respective initial values are the zeros of the given polynomial.

To identify the circumstances under which the slowest convergence might be expected, it is relevant to note that, with $z=x+i y$ and $\zeta$ given by (13),

$$
\left|\zeta-\frac{x}{x-1}\right|=\frac{1}{|x-1|}
$$

is independent of $y$, and

$$
\left|\zeta-\frac{y-i}{y}\right|=\frac{1}{|y|}
$$

is independent of $x[7$, p. 13, No. 4.4]. Thus, when $x \neq 1$ is held constant, and $y$ varies, the point $\zeta$ describes the circle (15), with center $x /(x-1)$ and radius $|x-1|^{-1}$. When $y$ is held constant, and $x$ varies, the point $\zeta$ describes the circle (16), with center $1-i / y$ and radius $|y|^{-1}$. Rough sketches of these circles, and of the unit circle $|\xi|=1$, show that the slowest convergence may be expected when a comparatively large value of $\left|y_{0}\right|$ is associated with a small value of $\left|x_{0}\right|$.

6. Numerical Examples. The polynomial $P_{8}(z)=z^{8}+20.4 z^{7}+151.3 z^{6}+$ $490 z^{5}+687 z^{4}+719 z^{3}+150 z^{2}+109 z+6.87$ is quoted by Scarborough [13, Example 2, p. 253], where the equation $P_{8}(z)=0$ is solved by root-squaring. In the course of this solution, the following results are obtained:

$\begin{array}{llll}A_{0} & 1.0 & 1.0 & 1.0 \\ A_{1} & 20.4 & -1.1 \times 10^{2} & -4.3 \times 10^{3} \\ A_{2} & 151.3 & 4.3 \times 10^{3} & 4.0 \times 10^{6} \\ A_{3} & 490 & -6.1 \times 10^{4} & -5.5 \times 10^{4} \\ A_{4} & 687 & -1.9 \times 10^{5} & -1.5 \times 10^{10} \\ A_{5} & 719 & -4.2 \times 10^{5} & -1.3 \times 10^{11} \\ A_{6} & 150 & -1.2 \times 10^{5} & 7.4 \times 10^{9} \\ A_{7} & 109 & -9.8 \times 10^{3} & -1.1 \times 10^{8} \\ A_{8} & 6.87 & 4.7 \times 10 & 2.2 \times 10^{3}\end{array}$

The coefficients of the original polynomial do not satisfy the termination criterion (7) for any value of $p$. The coefficients of the first and second iterates do, however, with $p=5$, indicating that $P_{8}(z)$ has five zeros outside the unit circle. This is confirmed by the computation of the zeros, which gives the values $z_{1}=-7.78, z_{2,3}=$ $-5.604 \pm 1.891 i, z_{4,5}=-0.6678 \pm 1.322 i, z_{6,7}=0.002818 \pm 0.413 i, z_{8}=-0.0674$. Since five applications of root-squaring, plus further computation, were required to obtain these values, it is realistic to state that the solution of the second stability problem for this polynomial, which is completed after the first root-squaring, is avail- 
able with significantly less effort than is required to evaluate its zeros.

This example, plus the general fact that the root-squaring procedure (6) ordinarily produces either very large or very small numbers, suggests the necessity of employing some type of scaling with the resultant iteration. A simple scaling procedure, which generates no arithmetic truncation error, is the reduction of the floating point scale factor of each coefficient such that the scale factor of the largest coefficient is zero, after each root-squaring. This procedure will be applied in the iterations given below.

It is of interest to apply the resultant iteration to solve the first stability problem for $P_{8}(z)$. The matrix $\Gamma$ in this case is

$$
\Gamma=\left|\begin{array}{rrrrrrrrr}
1 & 8 & 28 & 56 & 70 & 56 & 28 & 8 & 1 \\
1 & 6 & 14 & 14 & 0 & -14 & -14 & -6 & -1 \\
1 & 4 & 4 & -4 & -10 & -4 & 4 & 4 & 1 \\
1 & 2 & -2 & -6 & 0 & 6 & 2 & -2 & -1 \\
1 & 0 & -4 & 0 & 6 & 0 & -4 & 0 & 1 \\
1 & -2 & -2 & 6 & 0 & -6 & 2 & 2 & -1 \\
1 & -4 & 4 & 4 & -10 & 4 & 4 & -4 & 1 \\
1 & -6 & 14 & -14 & 0 & 14 & -14 & 6 & -1 \\
1 & -8 & 28 & -56 & 70 & -56 & 28 & -8 & 1
\end{array}\right|,
$$

whence $A_{0}=2.33457, A_{1}=-1.03136, A_{2}=-1.92884, A_{3}=-0.20032, A_{4}=1.6599$, $A_{5}=-0.46752, A_{6}=-0.71604, A_{7}=0.94784, A_{8}=-0.34223$, apart from inessential scale factors. These coefficients do not satisfy the termination condition (7) for any value of $p$. After eight iterations, $A_{0}=3.2 \times 10^{-1}, A_{1}=-2.0, A_{2}=3.7$, and the remaining coefficients are less than $10^{-10}$. The termination criterion (7) is thus satisfied with $p=2$, indicating that $P_{8}(z)$ has two zeros, $z_{6,7}$ in the right halfplane. The comparatively slow convergence of the resultant iteration is doubtless due to the particular numerical values $z_{6,7}=0.002818 \pm 0.413 i$ of these zeros.

It has been shown [5, pp. 74-76] that, as a consequence of arithmetic truncation error generated by the root-squaring procedure, the zeros of the successive resultants do not always correspond closely to the zeros of the original polynomial. The danger, in the present context, is that this lack of correspondence will be so substantial that zeros will, effectively, cross the unit circle, or imaginary axis, and give false information with respect to the original polynomial. While this possibility cannot be absolutely denied, it does appear that the repulsive nature of the points of these loci, which separate the regions of interest in stability calculations, is effective in preventing any iterate from crossing them. Thus, in the elementary example $[15, \mathrm{p} .74]$, the true zeros \pm 0.96950824 of the quadratic $0.93254613 z^{2}-0.12346723 \times 10^{-8} z-$ 0.87654321 correspond to the zeros 0.96962413 and -0.96939237 obtained from the first iterate, and one of these is, indeed, larger in absolute value than its correspondent. It is still within the unit circle and, after four further iterations using 10-digit (pocket calculator) arithmetic, the coefficients $1.07,0.79,0.15$ are obtained, which satisfy the termination criterion (7) with $p=0$, showing, correctly, that no zeros of 
the original polynomial lie outside the unit circle.

This same conclusion is justified by the more complicated examples cited. The polynomial $P_{16}(z)$ (loc. cit. p. 74) has six zeros within the unit circle and ten without. The largest modulus of a zero within the unit circle is 0.79 , the smallest modulus of a zero without is 1.06 . After three iterations, the polynomial obtained also has six zeros within the unit circle and ten without, although they do not correspond well with the zeros of $P_{16}(z)$. However, the largest modulus of a zero within the unit circle is now 0.15 , and the smallest modulus of a zero without is 1.65 , so it would appear that, despite the lack of correspondence in terms of numerical values, the solution of the second stability problem is proceeding correctly.

Finally, the polynomial $Q_{16}(z)=1250162561 z^{16}+385455882 z^{15}+$ $845947696 z^{14}+240775148 z^{13}+247926664 z^{12}+64249356 z^{11}+41018752 z^{10}+$ $9490840 z^{9}+4178260 z^{8}+837860 z^{7}+267232 z^{6}+44184 z^{5}+10416 z^{4}+1288 z^{3}$ $+224 z^{2}+16 z+2$ is reported [11, p. 396] to be such that "the root-squaring process fails to determine all the moduli even if twenty figures are retained in the initial transformations". Two transformations, using 10-digit (pocket calculator) arithmetic produce values which round to $A_{0}=2.44, A_{1}=0.273, A_{2}=0.821, A_{3}=-0.128$, $A_{4}=0.028$; remaining coefficients are negligibly small. It is seen that the second stability problem is solved, correctly, at this stage, with the conclusion that $p=0$, so that all the zeros lie within the unit circle.

Department of Mathematics

University of Ottawa

Ottawa, Ontario, Canada K1N 6N5

1. E. H. BAREISS, Resultant procedure and the mechanization of the Graeffe process,"

J. Assoc. Comput. Mach., v. 7, 1960, pp. 346-386.

2. E. H. BAREISS, "The numerical solution of polynomial equations and the resultant procedures" in Mathematical Methods for Digital Computers, Vol. II (A. Ralston \& H. S. Wilf, Editors), Wiley, New York, 1967, pp. 185-214.

3. W. S. BURNSIDE \& A. W. PANTON, The Theory of Equations, Two volumes, Dover, New York, 1960.

4. A. COHN, "Über die Anzahl der Wurzeln einer algebraischen Gleichung in einem Kreise," Math. Z., v. 14, 1922 , pp. 110-148.

5. E. D. DENMAN \& A. N. BEAVERS, JR., "The matrix sign function and computation in systems," Appl. Math. Comput., v. 2, 1976, pp. 63-94.

6. R. J. DUFFIN, “Algorithms for classical stability problems," SIAM Rev., v. 11, 1969, pp. 196-213.

7. H. KOBER, Dictionary of Conformal Representations, Dover, New York, 1952.

8. C. LANCZOS, Applied Analysis, Prentice-Hall, Englewood Cliffs, N. J., 1956.

9. M. MARDEN, Geometry of Polynomials, Math. Surveys, no. 3, Amer. Math. Soc., Providence, R. I., 1966.

10. D.-E. MAYER, "Sur les équations algébriques," Ann. de Math. (3), v. 10, 1891, pp. $111-124$.

11. F. W. J. OLVER, "The evaluation of zeros of high-degree polynomials," Philos. Trans. Roy. Soc. London Ser. A, v. 244, 1952, pp. 385-415.

12. J. PELTIER, Résolution Numérique des Équations Algébriques, Gauthier-Villars, Paris, 1957.

13. J. B. SCARBOROUGH, Numerical Mathematical Analysis, 6th ed., The Johns Hopkins Press, Baltimore, Maryland, 1966.

14. B. L. VAN DER WAERDEN, Modern Algebra, Vol. I, Ungar, New York, 1949.

15. J. H. WILKINSON, Rounding Errors in Algebraic Processes, Prentice-Hall, Englewood

Cliffs, N. J., 1963. 